\title{
Minimally Invasive Mitral Valve Surgery for Rheumatic Valve Disease
}

\author{
Anh Tuan Vo, MD, Khoi Minh Le, MD, Trang Thu Nguyen, MD, Thien Tam Vu, MD, \\ Chuong Tran Viet Pham, MD, Huy Quoc Tuan Ngo, MD, Tri Quang Le, MD, Dinh Hoang Nguyen, MD \\ Department of Cardiovascular Surgery, University Medical Center, University of Medicine and Pharmacy, Ho Chi Minh City, Vietnam
}

\section{ABSTRACT}

Background: The development of minimally invasive mitral valve surgery has created the motivation for using this approach in young patients with chronic rheumatic valve disease. We report our recent experience with patients undergoing minimally mitral valve surgery in this group of patients.

Methods: Between July 2014 and June 2018, 142 patients with rheumatic mitral valve dysfunction underwent minimally invasive surgery through a right thoracotomy approach at the University Medical Center of Ho Chi Minh City in Vietnam. Diagnosis was confirmed with transthoracic and transesophageal echocardiography (TTE and TEE). We analyzed the inhospital and midterm follow-up outcomes of this group.

Results: The mean age was $42.6 \pm 9.6$ years. Sixty patients $(42.3 \%)$ were male. Sixty-three patients were diagnosed with functional severe tricuspid regurgitation, 29 patients were identified with moderate tricuspid regurgitation, and tricuspid annulus was more than $\left.21 \mathrm{~mm} / \mathrm{m}^{2}\right)$. Mitral valve repair was performed in 16 patients $(11.3 \%)$, and 126 patients underwent mitral valve replacement. Mitral valve repair techniques included annuloplasty, leaflet peeling, and commissurotomy. Thirty-day mortality was $0.7 \%$. Two patients had to be converted to conventional sternotomy, due to left atrial appendage laceration and mitral annular rupture. The overall survival rate was $98.6 \%$. Freedom from reoperation was $97.1 \%$.

Conclusions: In patients with rheumatic valve disease, minimally invasive mitral surgery safely and effectively can be performed with few perioperative complications and good midterm results.

\section{INTRODUCTION}

The enthusiasm to perform minimally invasive surgery emerged in the last decade of the 20th century, due to successful laparoscopic and thoracoscopic procedures. Over the past two decades, this type of surgery has been advocated for many types of cardiac malformation and dysfunction, particularly for mitral valve disease. To decrease costs related to hospital stay and improve quality of life and cosmesis, consensus

Received April 27, 2019; accepted fuly 25, 2019.

Correspondence: Dinh Hoang Nguyen, 5 Dong Tien Apartment, District 10, Ho Chi Minh City, Vietnam; +84909500090 (e-mail: nguyenhoangdinb@yahoo.com). has been made that minimally invasive surgery provides greater satisfaction with patients while maintaining the safety and quality standard of a full sternotomy approach for mitral valve surgery.

Minimally invasive mitral valve surgery can be performed concomitantly with other procedures that include maze procedure, atrial septal defect closure, or tricuspid valve repair. In developing countries, chronic rheumatic mitral valve disease and secondary functional tricuspid valve regurgitation remains a major health problem. It is the most severe sequelae of rheumatic fever and occurs in approximately $30 \%$ of patients with rheumatic fever [WHO 2004]. The average annual incidence of acute rheumatic fever in children aged 5 to 15 years is $15.2 / 100,000$ population in Fiji [Steer 2009] compared with 3.4 cases/100,000 population in New Zealand [Jaine 2008] and less than 1 case/100,000 population in the United States.

The American Heart Association/ American Cardiology College's 2014 guidelines recommend tricuspid valve surgery for patients with severe tricuspid regurgitation undergoing left-sided valve surgery (Class I, Level C indication), and tricuspid valve repair can be beneficial for patients with mild, moderate, or greater functional TR (stage B) at the time of left-sided valve surgery with either tricuspid annular dilation ( $\geq 40 \mathrm{~mm}$ or $\geq 21 \mathrm{~mm} / \mathrm{m}^{2}$ ) or prior evidence of right HF (Class IIA, Level B indication) [Nishimura 2014].

Since patients with rheumatic mitral valve dysfunction tend to develop late functional tricuspid regurgitation [Pfannmuller 2013], there is a tendency to repair the tricuspid valve as a prophylactic procedure.

Presently, the minimally invasive approach for mitral valve surgery and concomitant procedures is being used in many

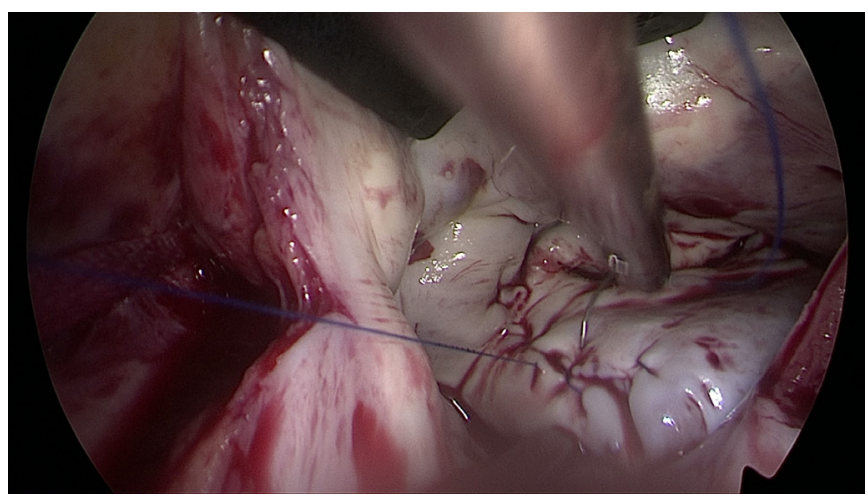

Figure 1. Left atrial appendage closure. 


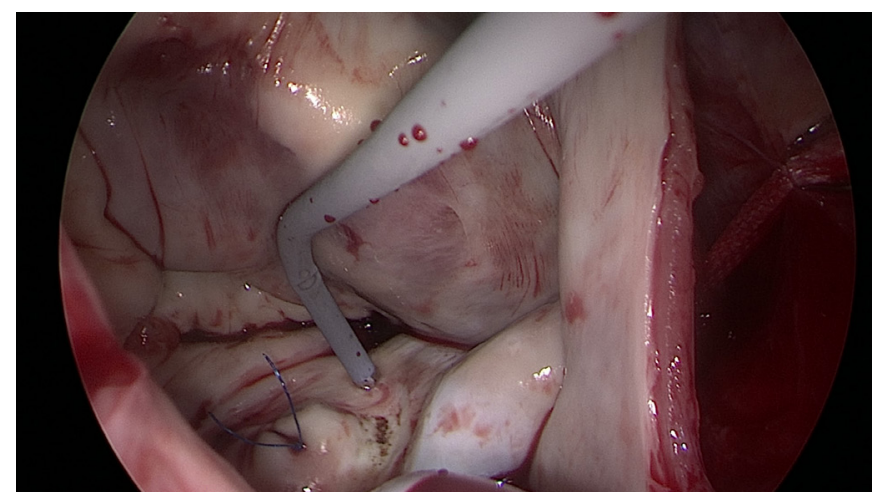

Figure 2. Concomitant Maze procedures.

centers with good short-term results and acceptable longterm results [Pfannmuller 2013; Seeburger 2008]. It also has proven to be as good and safe as the standard sternotomy approach even in elderly patients. In this report, we present our experience in minimally invasive mitral valve surgery in chronic rheumatic valve patients.

Patients and methods: Between July 2014 and June 2018, 142 patients with rheumatic valve disease underwent minimally invasive mitral surgery via a right thoracotomy approach at the University Medical Center, University of Medicine and Pharmacy at Ho Chi Minh City in Vietnam. Those who did not undergo minimally invasive through the right thoracotomy approach included patients who already had received previous surgery in the right thorax, patients with extensive mitral annular calcification, or patients with extensive mitral valve endocarditis requiring a complex reconstruction. Our primary outcome was in-hospital mortality and postoperative complications and midterm freedom from reoperation.

Preoperative, intraoperative, and postoperative clinical information was collected and analyzed. Preoperative and pre-discharge echocardiography also were recorded. The severity of valvular insufficiency was graded following the European Society of Cardiology (ESC) guidelines on the management of valvular heart disease.

Surgical technique: The patient was placed in the supine position with a cushion under the right scapula to facilitate exposure of both atria. A single lumen endotracheal tube intubation was used. We did not routinely apply single lung ventilation. With patients who weighed more than 75 kilograms or patients with concomitant ostium secundum atrial septal defect, an additional right jugular venous cannula was inserted percutaneously by the surgeon and anesthetist following the induction of anesthesia. Cardiopulmonary bypass $(\mathrm{CPB})$ was achieved with cannulation of the femoral artery and vein through a small incision in the right groin. We routinely used the bicaval venous cannula if the jugular vein cannula was not necessary. Transesophageal echocardiography (TEE) was used to confirm the ideal position of the guidewire and the tip of the venous cannula in the superior vena cava. If severe peripheral vascular disease or high-grade aortic atheroma was present, we used axillary cannulation and avoided retrograde perfusion. CPB was initiated using a closed

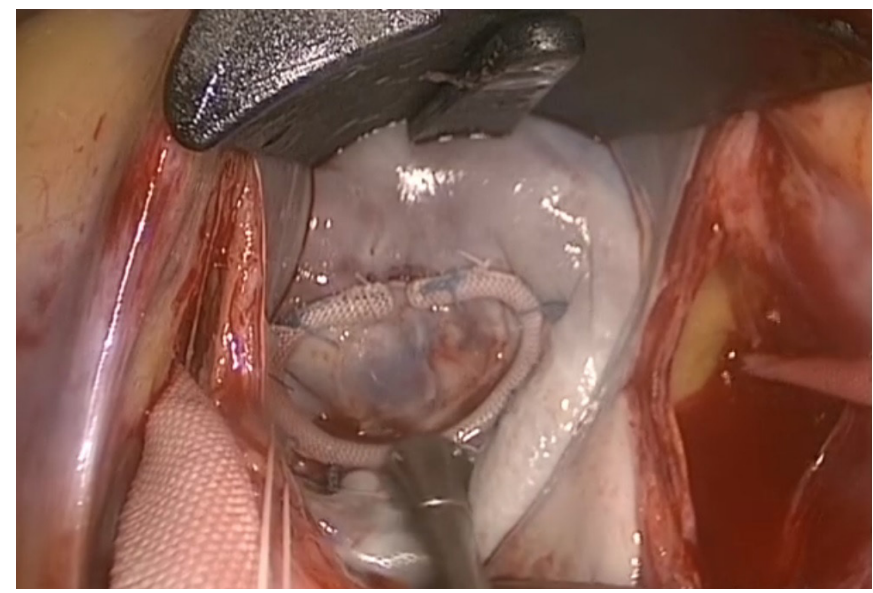

Figure 3. Rheumatic valve repair showing a competent non stenotic mitral valve.

membrane oxygenator and roller pump. Rectal temperature was maintained at $32^{\circ} \mathrm{C}$ and vacuum-assisted venous drainage was utilized during the procedure with the negative pressure ranging around $-50 \mathrm{mmHg}$ to decompress the heart.

For the right minithoracotomy, we applied the method of Lamelas to decide the exact site of the thoracotomy incision [Lamelas 2015]. The sternum was marked from the suprasternal notch to the lowest point of the xiphoid. Subsequently, the midpoint of the entire sternum was located and a line was drawn from this point laterally. A 4-5 cm skin incision was made parallel to and starting at the anterior axillary line. This usually corresponds with the fourth intercostal space and provides excellent exposure for both the mitral and tricuspid valves. When trying to decide whether the intercostal space was high enough, when the right pleural space was entered, we used a finger to touch the diaphragm. If this structure could not be felt, the intercostal space was suitable. If the surgeon touches the diaphragm, it is advised to go one intercostal space higher. A dedicated instrument set designed for minimally invasive surgery was used to operate (Aesculap Inc., Pennsylvania, U.S.A). A soft tissue retractor and mini thoracic retractor were used to spread the ribs. A video camera was inserted through a $5 \mathrm{~mm}$ port in the third right intercostal space. The pericardium was opened $3 \mathrm{~cm}$ anterior, parallel to the phrenic nerve and extended from the distal ascending aorta to the diaphragm. Several traction sutures were placed. We routinely used 3 stitches at the cardiophrenic angle. These sutures were pulled out through a small incision two intercostal spaces lower than the primary incision, and this provided better exposure by caudally pulling the diaphragm. The oblique sinus bluntly was dissected, and the inferior vena cava occlusion tape was passed at that time. This snare also was pulled out at the same level as the diaphragmatic traction sutures. Another snare was placed around the superior vena cava anteriorly to the right pulmonary artery. The transthoracic Chitwood aortic cross-clamp was inserted via a $5 \mathrm{~mm}$ incision in the third right ICS. Care was taken when clamping the aorta to avoid left atrial appendage laceration. Two liters of Custodiol HTK solution $(30-50 \mathrm{~mL} / \mathrm{kg}$ ) 


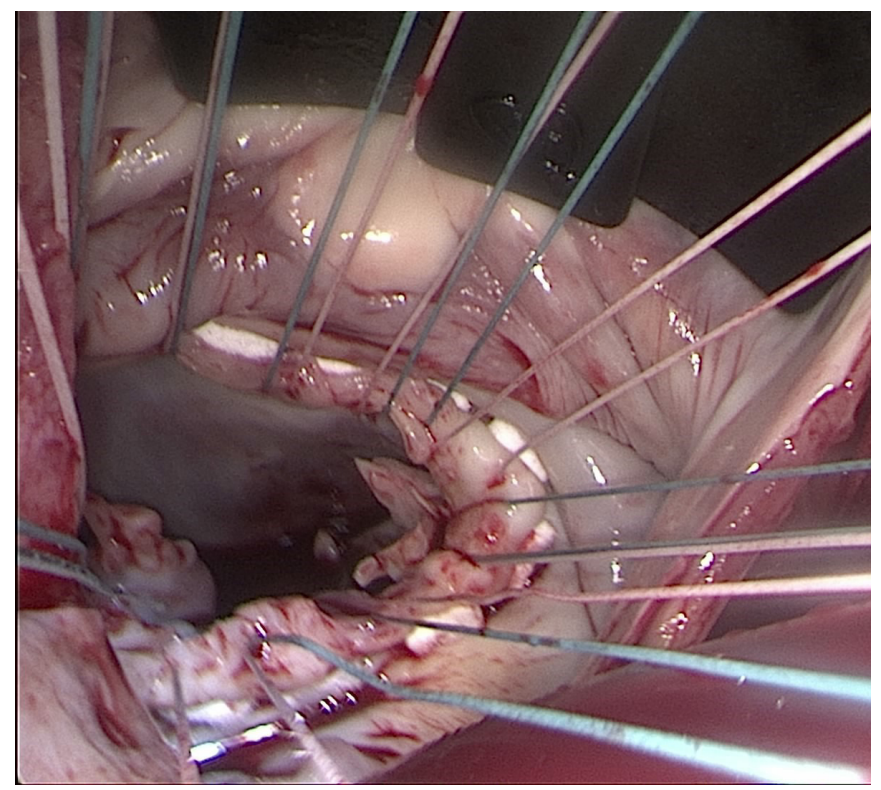

Figure 4. Mattress pledgeted sutures with preservation of posterior leaflet.

was delivered antegrade into the aortic root through a long metal cardioplegia needle and repeated every 120 minutes, if necessary. A left atriotomy was performed and sump suction inserted to keep the surgical field cleared. A left atrial retractor was used to expose the mitral valve. Concomitant Maze lesions were made in the left atrium with a long monopolar probe (Medtronic, Minneapolis, MN, USA), and left appendage closure was performed in patients with chronic atrial fibrillation (Figures 1 and 2).

We then assessed the mitral valve for the feasibility of repair. Repairing the post rheumatic valve was challenging, applicable techniques were leaflet peeling and commissurotomy. A classic rigid annuloplasty ring was implanted to support the repair (Figure 3 ).

If the valve heavily was damaged and repair not achievable, mitral valve replacement was performed with horizontal mattress sutures. Pledgets were used in the case of annular calcification. We routinely preserved the posterior leaflet and, in most cases, some parts of anterior leaflet chordae, and reattached them to the annular sutures to reduce the risk of postoperative left ventricle remodeling [Rozich 1992]. Carbon dioxide continuously was infused via the atrial traction system during the surgery. After the mitral valve procedure, the left atriotomy was closed using a 3.0 Prolene suture with the sump suction left in the left ventricle through the mitral valve (Figures 4 and 5).

Both vena cava snares securely were snugged and a right atriotomy was performed from the base of the right atrial appendage obliquely via the left atriotomy. A small left atrial retractor was used to expose the tricuspid valve. We performed the annuloplasty with a tricuspid rigid ring (Carpentier - Edwards ring, Edwards Lifesciences, Irvine, CA, U.S.), or an autologous pericardial band. Kay suture or De Vega technique no longer was used in our center.

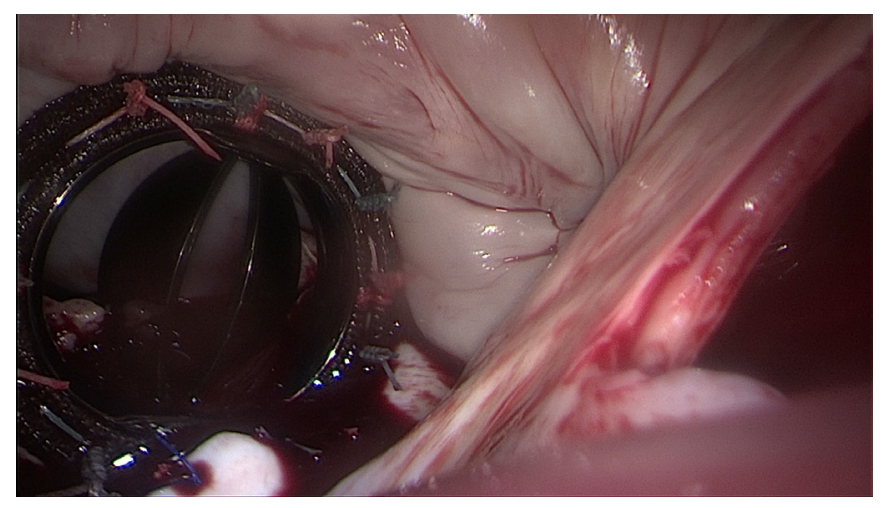

Figure 5. Mechanical valve replacement.

Tricuspid valve repair also could be performed after declamping the aorta if the cardiac arrest time was more than 120 minutes. The right atriotomy was closed in a double-layered fashion with a 5.0 Prolene suture. The patient was placed in the Trendelenburg position, and the lungs ventilated to facilitate deairing. The aortic clamp then was removed and cardiopulmonary bypass slowly was weaned to allow assessment of the mitral and tricuspid valve by TEE. Subsequently, CPB was restarted for hemostasis. The patient then was weaned off extracorporeal circulation and decannulated. One chest tube and one silicon drain were placed in the right pleural cavity and pericardium.

Follow-up: All patients were reassessed with transthoracic echocardiography (TTE) at the outpatient consultation at 1 month, 6 months, 12 months, and every 6 months, postoperatively. The mean follow-up time was 25.8 months.

\section{RESULTS}

Demographic characteristics: Between July 2014 to June 2018, a total of 142 patients with rheumatic valvular disease underwent minimally invasive mitral surgery. Sixty patients $(42.3 \%)$ were male; the mean body mass index (BMI) was $22.8 \pm 4.4$. Mean EuroSCORE II was $1.9 \pm 0.41$. The average NYHA was $2.1 \pm 0.32$. Most patients had good left ventricle function, mean left ventricular ejection fraction was $65.3 \%$, atrial fibrillation was present in 26 patients. Concomitant Maze procedure was performed in 19 patients with a left atrial (LA) diameter less than $60 \mathrm{~mm}$ and LA volume $<150 \mathrm{~mL}$. Sixty-three patients were diagnosed with functional severe tricuspid regurgitation; the other 29 patients were identified with moderate tricuspid regurgitation, but tricuspid annulus was more than $21 \mathrm{~mm} / \mathrm{m}^{2}$. There were 39 patients with pure mitral stenosis, 12 patients with pure mitral regurgitation, and 91 patients with mitral stenosis and regurgitation.

Mean cardiopulmonary bypass time was $132.2 \pm 41.9 \mathrm{~min}-$ utes, aortic cross-clamp time was $82.3 \pm 27.1$ minutes.

Mitral valve replacement was performed in 126 patients, and 16 other patients underwent mitral valve repair. One patient needed to replace the valve, due to unsatisfactory repair on transesophageal echocardiography. Tricuspid valve repair was carried out in 92 patients, and 16 patients 
Table 1. Repair techniques for tricuspid valve

\begin{tabular}{ll}
\hline Techniques & $\mathrm{N}=28$ \\
\hline Autologous pericardial band & $9(32.1 \%)$ \\
Classic rigid ring & $19(67.9 \%)$
\end{tabular}

received an autologous pericardial band while a rigid ring (Carpentier - Edwards Classic tricuspid ring, Edwards Lifesciences, Irvine, CA, U.S.) was implanted in the other 76 patients.

Early outcomes: Thirty-day mortality was $0.7 \%$. One patient died due to nosocomial pneumonia after stroke that require prolonged mechanical ventilation. Two patients required conversion to sternotomy - one due to left atrial appendage laceration caused by the Chitwood clamp, and the other due to mitral annular rupture. There was one patient with permanent stroke $(0.7 \%)$. Two patients had a transient decrease in renal function, which improved after 2 days in the ICU. Seven patients were reoperated for bleeding and hemothorax. No aortic dissections were recorded. Four patients developed atrial fibrillation postoperatively (no preoperative atrial fibrillation) and successfully were converted to sinus rhythm with Amiodarone in the ICU. The mean mechanical ventilation time was 9.8 hours 2.9 hours. Mean ICU time was 1.6 days and mean ward stay was 6.7 days. Conversion to sinus rhythm was achieved in $18 / 19$ patients who underwent concomitant Maze procedure. One patient (5.2\%) developed Mobitz II second degree AV block and required a permanent pacemaker.

Overall mortality after 47 months followed up was $1.41 \%$ ( 2 patients). The other patient died because of cerebral hemorrhage related to anticoagulation.

Mitral valve repair: Out of 142 patients, only 15 patients successfully were repaired (10.6\%), and one patient required intraoperative mechanical valve replacement due to persistent high gradient. Repair techniques include leaflet peeling and commissurotomy. Transesophageal echocardiography was used to check the effectiveness of the procedure. Before discharge, one transthoracic echocardiography was performed to confirm the result of mitral valve repair. Three other transthoracic echocardiographic also were done by cardiologists to reassess the mitral valve at 1 month, 6 months, 12 months, and every 6 months after showing that residual mitral regurgitation was less than moderate in only 2 patients. The mean transmitral gradient after 12-month follow up was $3.6 \mathrm{mmHg}$ in this group.

Mitral valve replacement: Mitral valve replacement was performed in 126 patients. Mechanical valves were implanted in 121 patients. Bioprosthetic valves were chosen for young females, who wished to have children (4 patients) or patients who refused long-term anticoagulation (1 patient).

Two-year freedom from operation was $97.1 \%$. Three patients with mechanical valves were reoperated via full sternotomy, due to valve dysfunction at 12 months, 19 months, and 26 months postoperative, respectively.

Tricuspid valve repair: Autologous pericardial band or a rigid ring was used for the tricuspid valve repair. Kay suture and De Vega techniques no longer were applied in our center (Tables 1 and 2).

\section{DISCUSSION}

Minimally invasive mitral valve surgery in chronic rheumatic valve disease is safe and feasible. Operation time, cardiopulmonary bypass and cross-clamp time were satisfactory, associated with short hospital stay, and displayed good shortterm results. This showed that a minimally invasive approach is a good substitute for the conventional median sternotomy.

Current evidence suggests that minimally invasive mitral surgery is associated with decreased blood loss, less blood product transfusion, atrial fibrillation, sternal wound infection, scar dissatisfaction, decreased ventilation time, intensive care unit stay, hospital length of stay and reduced time to return to normal activity, with no adverse impact on longterm need for valve reoperation and survival beyond 1 year [Cheng 2011; Ding 2014]. In 2010, the consensus statement of the International Society for Minimally Invasive Cardiothoracic Surgery (ISMICS) also showed that minimally invasive mitral surgery had comparable short-term and long-term mortality. Our results confirmed the same efficiency and safety for patients undergoing minimally invasive mitral surgery for rheumatic heart disease.

However, there remain debates regarding a minimally invasive approach for mitral valve surgery, despite good outcomes in both short- and long-terms, including the increased risk of neurological event, higher rate of valve replacement over valve repair, aortic dissection, and groin infection [Cheng 2011]. Among these concerns, permanent stroke was one of the severe complications, which increased mortality and seriously affected the patient's quality of life. Some authors suggested that retrograde perfusion and endoaortic clamping were the main risks for postoperative neurological complications, particularly in patients with severe atherosclerotic vascular disease [Modi 2013; Murzi 2013; Luca 2013]. In our study, there was one patient with permanent stroke after surgery. We did not apply an endoclamp but used a transthoracic aortic Chitwood clamp. The patient was a 54-year-old smoker. We assumed the reason for the stroke was peripheral cardiopulmonary bypass on a high-risk vascular disease patient; the patient died due to nosocomial pneumonia. After this event, we routinely performed a thoracoabdominal computed tomography (CT) scan in patients over 45 years, with at least one risk factor for cardiovascular disease to assess the risk of embolism. If there was any high-risk atherosclerotic plaque (grade IV/V) [Sharma 2011] on the aorta, we changed the approach to axillary cannulation. This provided an antegrade flow and could decrease the risk of embolism [Luca 2013; Glauber 2015].

Rheumatic valve was still dominant in our population. Because of the morphology and evolution of this type of disease, mitral replacement usually is a choice for cardiac surgeons. However, several authors have published a positive result of rheumatic valve repair with better short-term and long-term event-free survival [Schaff 2015; Wang 2013; 
Table 2. Residual tricuspid regurgitation

\begin{tabular}{lcccc}
\hline Rate of regurgitation & At discharge $(\mathrm{N}=28)$ & 1 month $(\mathrm{N}=28)$ & 6 month $(\mathrm{N}=28)$ & 1 year $(\mathrm{N}=28)$ \\
\hline No regurgitation & 16 & 17 & 3 & 17 \\
Trivial & 4 & 3 & 7 & 3 \\
Mild & 8 & 7 & 1 & 7 \\
Moderate & 0 & 1 & 0 & 0 \\
Severe & 0 & 0 &
\end{tabular}

Chotivatanapong 2012]. Sixteen patients with rheumatic mitral valve stenosis underwent valve repair in our series. We did a commissurotomy and peeled the leaflet to effectively remove the calcium deposits and fibrotic tissue on the leaflet, thus providing better flexibility of the valve. Also, papillary muscles were split to their base to provide length for the leaflets. A classic rigid ring was implanted to reinforce the mitral annulus. The mean gradient through the mitral valve after a 12 -month follow-up was $3.6 \mathrm{mmHg}$. Thus, we believe complex repair techniques for repairing a rheumatic valve using conventional sternotomy also could be used in a minimally invasive approach through the right minithoracotomy.

In our center, we followed guidelines and recommendations for tricuspid valve repair and concomitant tricuspid valve repair. Mitral valve surgery was indicated for patients with dilated tricuspid valve annulus on transthoracic echocardiography in the four-chamber view. However, with Asian populations, a specific number does not raise a good indication, thus we used criteria based on BMI to decide when to repair the tricuspid valve. According to Chopra et al [Chopra 1989], one should repair the valve if the annular diameter is $21 \mathrm{~mm} / \mathrm{m}^{2}$ ). There were 16 patients implanted with an autologous pericardial band. We used this type of repair technique in patients with moderately dilated tricuspid regurgitation to decrease the cost of the surgery. However, in patients with severe tricuspid regurgitation and a dilated annulus (i.e $40 \mathrm{~mm}$ ), we routinely used a classic rigid ring to ensure the long-term result. One patient had worsened tricuspid residual regurgitation from mild to moderate. This patient was implanted with a rigid ring with a very large right atrium and tricuspid annulus. We counted this patient as a failure in tricuspid repair and continue following up with this valve.

Finally, regarding the learning curve for mitral valve repair in combination with tricuspid valve repair, a cross-sectional survey concluded that at least 20 cases are required to gain familiarity with the minimally invasive approach for the mitral valve and at least one case needed to be performed per week to maintain proficiency [Misfeld 2013]. Another author suggested the typical number of operations to overcome the learning curve was between 75 and 125. Since 2014, minimally invasive has become our standard approach for treatment of lesions in the atria, including mitral valve disease, tricuspid valve disease, atrial myxoma, and some congenital heart diseases (atrial septal defect, atrioventricular canal, and perimembranous ventricular septal defect in the adults). In our experience, the number required to pass the learning curve for this approach should be more than 50 cases. Also, the surgeon should be acquainted and confident with the conventional sternotomy before starting with minimally invasive surgery.

The main limitation of this study was the small number of patients and short-term follow up. However, we reported early good results of mitral valve surgery in combination with tricuspid valve repair, particularly the promising outcome for rheumatic mitral valve repair with complex techniques proving the feasibility of facing this challenging disease.

\section{CONCLUSION}

In conclusion, minimally invasive mitral surgery in rheumatic valve disease is feasible and a safe alternative to conventional sternotomy surgery. The latter should be reserved for complex and severe patients. This approach is associated with low mortality and morbidity, and a promising successful repair rate.

\section{REFERENCES}

Cheng DC, Martin J, Lal A, Diegeler A, Folliguet TA, Nifong LW, et al. 2011. Minimally invasive versus conventional open mitral valve surgery: a meta-analysis and systematic review. Innovations (Phila). 6(2):84-103.

Chopra HK, Nanda NC, Fan P, Kapur KK, Goyal R, Daruwalla D, et al. 1989. Can two-dimensional echocardiography and Doppler color flow mapping identify the need for tricuspid valve repair? J Am Coll Cardiol. 14(5):1266-74.

Chotivatanapong T, Lerdsomboon P, Sungkahapong V. 2012. Rheumatic mitral valve repair: experience of 221 cases from Central Chest Institute of Thailand. J Med Assoc Thai. 95 Suppl 8:S51-7.

Ding C, Jiang DM, Tao KY, Duan QJ, Li J, Kong MJ, et al. 2014. Anterolateral minithoracotomy versus median sternotomy for mitral valve disease: a meta-analysis. J Zhejiang Univ Sci B. 15(6):522-32.

Glauber M, Miceli A, Canarutto D, Lio A, Murzi M, Gilmanov D, et al. 2015. Early and long-term outcomes of minimally invasive mitral valve surgery through right minithoracotomy: a 10-year experience in 1604 patients. J Cardiothorac Surg. 10:181.

Jaine R, Baker M, Venugopal K. 2008. Epidemiology of acute rheumatic fever in New Zealand 1996-2005. J Paediatr Child Health. 44(10):564-71.

Lamelas J. 2015. Concomitant minithoracotomy aortic and mitral valve surgery: the minimally invasive "Miami Method". Ann Cardiothorac Surg. 4(1):85-7. 
Luca F, van Garsse L, Rao CM, Parise O, La Meir M, Puntrello C, et al. 2013. Minimally invasive mitral valve surgery: a systematic review. Minim Invasive Surg. 179569.

Misfeld M, Borger M, Byrne JG, Chitwood WR, Cohn L, Galloway A, et al. 2013. Cross-sectional survey on minimally invasive mitral valve surgery. Ann Cardiothorac Surg. 2(6):733-8.

Modi P, Chitwood WR, Jr. 2013. Retrograde femoral arterial perfusion and stroke risk during minimally invasive mitral valve surgery: is there cause for concern? Ann Cardiothorac Surg. 2(6):E1.

Murzi M, Cerillo AG, Miceli A, Bevilacqua S, Kallushi E, Farneti P, et al. 2013. Antegrade and retrograde arterial perfusion strategy in minimally invasive mitral-valve surgery: a propensity score analysis on 1280 patients. Eur J Cardiothorac Surg. 43(6):e167-72.

Nishimura RA, Otto CM, Bonow RO, Carabello BA, Erwin JP, 3rd, Guyton RA, et al. 2014. 2014 AHA/ACC guideline for the management of patients with valvular heart disease: a report of the American College of Cardiology/American Heart Association Task Force on Practice Guidelines. J Thorac Cardiovasc Surg. 148(1):e1-e132.

Pfannmuller B, Davierwala P, Hirnle G, Borger MA, Misfeld M, Garbade J, et al. 2013. Concomitant tricuspid valve repair in patients with minimally invasive mitral valve surgery. Ann Cardiothorac Surg. 2(6):758-64.
Rozich JD, Carabello BA, Usher BW, Kratz JM, Bell AE, Zile MR. 1992. Mitral valve replacement with and without chordal preservation in patients with chronic mitral regurgitation. Mechanisms for differences in postoperative ejection performance. Circulation. 86(6):1718-26.

Schaff HV. 2015. Mitral valve repair in patients with rheumatic heart disease: what are the limits? J Thorac Cardiovasc Surg. 149(3):779-80.

Seeburger J, Borger MA, Falk V, Kuntze T, Czesla M, Walther T, et al. 2008. Minimal invasive mitral valve repair for mitral regurgitation: results of 1339 consecutive patients. Eur J Cardiothorac Surg. 34(4):760-5.

Sharma U, Tak T. 2011. Aortic atheromas: current knowledge and controversies: a brief review of the literature. Echocardiography. 28(10):1157-63.

Steer AC, Kado J, Jenney AW, Batzloff M, Waqatakirewa L, Mulholland EK, et al. 2009. Acute rheumatic fever and rheumatic heart disease in Fiji: prospective surveillance, 2005-2007. Med J Aust. 190(3):133-5.

Wang Z, Zhou C, Gu H, Zheng Z, Hu S. 2013. Mitral valve repair versus replacement in patients with rheumatic heart disease. J Heart Valve Dis. 22(3):333-9.

World Health Organ Tech Rep Ser. 2004. Rheumatic fever and rheumatic heart disease. 923:1-122, back cover. 